\title{
Analysis of Algebra Content in Mathematics Books in the Third Grade to the Fifth Grade in Jordan in Light of the Standards of the American National Council of Mathematics Teachers
}

\author{
Dr. Hamzeh Ahmad Abd-Alrohman Alqiam ${ }^{1}$ \\ ${ }^{1}$ Jerash University / Faculty of Educational Sciences, Jordan \\ Correspondence: Dr. Hamzeh Ahmad Abd-Alrohman Alqiam, Jerash University / Faculty of Educational \\ Sciences, Jordan. E-mail: m.alzboon@yahoo.com
}

Received: July 18, 2019

Accepted: August 4, 2019

Online Published: August 5, 2019

doi:10.5539/mas.v13n9p1

URL: https://doi.org/10.5539/mas.v13n9p1

\begin{abstract}
This study aimed at analyzing the content of algebra in mathematics books in the third grade to the fifth grade in Jordan in light of the standards of the National Council of Mathematics Teachers (NCTM, 2000), In all aspects of the study for the academic year (2018/2019). The sample of the study included the axis of algebra in each book of this stage. To achieve this, the descriptive method was used through the method of content analysis. Lycartes averages.

The highest scores in the third, fourth and fifth grade textbooks were for the criterion of analysis of change in different environments (1.84), while the lowest level of understanding of relations, patterns and functions was (0.90) And the use of mathematical models between these two grades. In light of these results, the researcher recommends that the criteria for the content of algebra should be considered in the basic stage books in the third, fourth and fifth grades.
\end{abstract}

Keywords: content analysis, content of algebra, standards

\section{Introduction}

Mathematics is one of the oldest and most advanced sciences in human history. It plays an important role in the life of the individual as it is indispensable in the organization of various daily activities. Therefore, it has developed and developed in all areas of life. To the level reached now, where mathematics has been in the service of the individual and community service since ancient times, has been used in the conduct of the daily life of individuals from agriculture, industry and Amran.

The objectives of teaching mathematics have evolved from a simple focus on precision and speed in computation to a focus on comprehension and problem solving, which is one of the basic objectives of mathematics teaching. Therefore, the ability of students to solve mathematical problems has occupied a large part of the interest of researchers in the field of mathematics teaching. (NIMSI) in Britain, and the National Council of Teachers of Mathematics (NCTM), USA (Abdelkader, 2013).

Mathematics is the field of Khasab for training in sound thinking through problem situations in which the relations between the elements are required to understand and plan for solving them. This view of mathematics imposes on its teachers because, in the same traditional way, the traditional mathematics curriculum offers little to build the personality of the student. This article needs a new approach and a new learning method (Al-Astal et al-Rashid, 2004).

Algebra is an important part of mathematics that connects it to the real world where its information is applied in all aspects of life. Algebra is one of the basic branches of mathematics, especially in the basic stage (Al-Sadik, 2001).

Algebra is an effective thinking tool that makes mathematical ideas more solid and helps students identify the common mathematical elements of different mathematical situations (Greeno \& Hall, 1997).

The National Council of Teachers of Mathematics emphasized the importance of algebra to understand mathematical relationships and concepts (NCTM, 2000). The importance of algebra also lies in involving students in the multiple activities of the single mathematical idea. 
He pointed out that throughout the ages, the pursuit of quality in the teaching of mathematics was that since the middle of the last century, educational movements and attitudes have emerged for the development of curricula. The movement of behavioral goals and then the movement of learning outcomes, then the culture of standards, In the development of standards that the learner must know since his youth in all stages of his educational career.

In this regard, the National Council of Teachers of Mathematics has issued many documents related to the development and teaching of mathematics, such as the Curriculum Standards and School Mathematics Evaluation Standards, the Professional Standards Document (NCTM, 1991), the School Mathematics Evaluation Standards (NCTM, 1995) And school mathematics standards (NCTM, 2000). These standards have certainly distinguished the standards of processes, content and intellectual skills, and have formed the basis for guiding the development of educational curricula.

Therefore, many studies have been conducted on the international standards of mathematics in many Arab and foreign countries, such as the Raba'a and Makadi study (2016), the study of Rammona et al. (2015) et al., 2010), the Marianne study (Mrayyan, 2013) and other studies that have been recommended to conduct many studies in the light of global standards.

The study of Rababa'a and Makdadi (2016) aimed to identify the extent to which the mathematics book developed for the first grade in Jordan included the standards of the National Council of Mathematics Teachers (NCTM). The researchers adopted the descriptive analytical approach. To achieve the objective of the study, the researchers developed a tool for content analysis in the light of the standards (NCTM, 2000). The results of the study showed that the content of the book includes $68 \%$ of the NCTM standards and that the field of statistics and possibilities has been overlooked and other areas are included in varying percentages.

The study of Rammna et al. (2015) aimed to analyze the content of the mathematics textbooks in the basic stage from the first to the fourth grade in Jordan in light of the standards of the National Council of Mathematical Teachers (NCTM, 2000). The researchers used the descriptive analytical approach. To achieve the goal of the study, the researchers used the content analysis tool, which comes from the standards of the NCTM. The study showed that the highest level of availability in the first four grades was for the standard of relationships and linkages while the lowest level of availability was for the communication standard.

The Mrayyan study (2013) aims to analyze the content of engineering in school mathematics textbooks from the first grade to the sixth grade in Jordan in light of the standards of the National Council of Mathematics Teachers (NCTM). The researcher adopted the descriptive analytical approach. To achieve the objective of the study, the researcher used a list of criteria for engineering that emanates from the standards of the National Council of Mathematics Teachers (NCTM) for all stages. The results showed that the content of engineering in Jordanian curricula does not comply with the standards of the National Council of Mathematics Teachers, where the average availability of standards in the curriculum content is 39.8 .

Therefore, this study was characterized by previous studies aimed at analyzing the content of algebra by the third, fourth and fifth grade mathematics textbooks in light of the standards of the National Council of Mathematics Teachers, and agreed with previous studies using the descriptive method through the method of content analysis.

In order to cope with the development and change, the Ministry of Education sought to develop the curricula and make them contemporary curricula that meet the needs and achieve the goals. Therefore, the Ministry has developed guidelines for the developed curricula. It began changing the textbooks for the basic stage and then applying them, the third, fourth and fifth grades were written in light of the NCTM's standards of comprehensiveness, realism and importance, in an effort to keep the books in line with international standards. Based on the above, the study problem is determined as follows:

\section{The Problem of the Study and Its Questions}

The researcher identified the problem of the study with the following main question:

"To what extent does algebra content in math books in the third grade guarantee the fifth grade of the National Council of Mathematics Teachers (NCTM, 2000)?"

It is divided into the following sub-questions:

What are the school mathematics standards issued by the National Council of Mathematics Teachers (NCTM, 2000) on the content of algebra in math books in the third grade to the fifth grade in Jordan?

What is the availability of school mathematics standards issued by the National Council of Mathematics Teachers (NCTM, 2000) on the content of algebra in math books in the third grade to the fifth grade in Jordan? 


\section{Objectives of the Study:}

The present study aims to achieve the following objectives:

Analysis of the content of Algebra mathematics mathematics books in the third row to grade to the fifth grade in Jordan in the light of NCTM standards.

Identify the extent to which the content of algebra in mathematics books in the third grade to the fifth grade of the basic standards of the National Council of Teachers of Mathematics NCTM.

Identify the standards that must be available in the content of Algebra mathematics books to meet the standards of NCTM.

\section{The Importance of Studying:}

The importance of the study is that:

This study comes as a response to global trends, which calls for analysis of the curriculum and evaluate and develop.

Provide a tool for content analysis in light of the standards of the National Council of Teachers of Mathematics NCTM.

May meet the needs of students of science and researchers and those interested in the analysis of curricula in light of the standards of the National Council of Teachers of Mathematics NCTM.

\section{Terminology of Study}

For the purposes of the study, the following terms shall be defined as follows:

Content Analysis: Al-Laqqani and Al-Majal (1986: 86) define it as "a method that is used in conjunction with other approaches to evaluate the curriculum for its development. It depends on the objectives of the analysis and the analysis unit to determine the prevalence of a phenomenon or concept or idea or more." "This is the method that aims to give a quantitative and objective description in the light of the NCTM standards to determine the compatibility of algebra content in the third, fourth, and fifth grade mathematics textbooks with those of the NCTM."

Algebra content: The concepts and skills included in the third, fourth, and fifth grade mathematics books.

Standards: Known by al-Lakani and Jamal $(2003,279)$ : "The views of many psychological, social, scientific and educational dimensions can, through their application, define the true picture of the object to be evaluated or reach judgments about what it is doing." The researcher defines it as a set of terms, conditions or specifications that we will build based on the NCTM standards and appears as a list in the light of which the algebra content of the mathematics books is analyzed from the third grade to the fifth grade.

The US National Council of Mathematics Teachers' Standards: A set of basic standards used in many countries of the world to build a mathematical curriculum (Abu Musa, 1997: 11).

\section{The Limits of the Study}

The results of this study are determined by the study population which consists of the following:

Analysis of algebra content in mathematics books in the third, fourth and fifth grades.

Analysis will be in accordance with the standards of the National Council of Teachers of Mathematics NCTM.

\section{Method and Procedures:}

A description of the study methodology, community and sample is provided. The study tool was also described and applied procedures, and how to find its validity and stability, as well as a description of the statistical treatments that were followed to answer the questions of the study.

\subsection{The Community and Study of the Study}

The study was conducted on the textbooks of school mathematics, which are scheduled for students of the basic stage of grades (3-5) for the academic year (2018/2019). The study sample included the content of algebra in each book of this stage.

\subsection{Study Methodology and Tools:}

The study used the descriptive method through the use of the method of content analysis, and used in this study to describe the content of algebra in the mathematics books of the basic stage to see the inclusion of the standards the National Council of Teachers of Mathematics (NCTM). 
After reviewing the standards of the US National Council (NCTM, 2000) through the third to fifth grade, a list of criteria for the algebra content of the basic mathematics textbooks (III to V) is presented. Table 1 shows these criteria, the list included 14 sub-criteria divided into four main criteria.

Table 1. List of content standards to be available in algebra content

\begin{tabular}{c} 
Axles \\
\hline Understanding \\
relationships, patterns and \\
functions \\
Representation and \\
analysis of mathematical \\
and structural positions \\
using algebraic symbols
\end{tabular}

Use mathematical models to represent and understand quantitative relationships.

Analyze change in different environments

\title{
Standards
}

\begin{abstract}
Describes generalizations about geometric and numerical patterns.
Determines generalizations about geometric and numerical patterns.

Be generalizations about geometric and numerical patterns.

Represent styles and functions using words, tables, and graphics.

Analyzes patterns and functions using words, tables, and graphics.
\end{abstract}

Characterizes algebraic properties such as substitution, assembly and distribution.

Uses properties such as substitution, assembly, and distribution in computations to total numbers.

The idea of the variable is represented as anonymous by using letters or algebraic symbols.

Express mathematical relationships using equations.

Mathematical problems are integrated into things.

Uses representations such as graphs, tables, and equations to derive results.

Investigate the effect of change in one variable on the second variable.

Distinguish mathematical positions using constants or variables and compare them.

Describe mathematical positions using constants or variables and compare them.

This list was used as a data analysis card according to the five-liqueur system, as follows:

- Very available $=4$

- Highly available $=3$

- Moderately available $=2$

- slightly available $=1$

- Not available $=0$

\subsection{Procedures for Analyzing Algebra Content}

The content analysis was conducted by five teachers of mathematics in the schools of the Hashemite Kingdom of Jordan, which were trained beforehand and before the study began on how to analyze the content. The procedures of analyzing the algebra content in mathematics books for the basic stage proceeded according to the following rules and procedures:

Conscious knowledge of the content of algebra in the book of each row separately.

Reading each subject (analysis unit) Read carefully and accurate several times.

Reading the list of content criteria (categories of analysis) in-depth conscious reading.

To consider each activity paragraph and each training or exercise or paragraph example.

Searching for the availability of the standard in each subject and its repetition in the paragraphs that were agreed upon by those who analyzed.

Place the mark (/) in the space allocated according to the receipt of the item of each of the content criteria specified in the list of analysis in the subject and repeat it according to the number of times this item of the criterion is received in the subject.

Unloading the results of the analysis of each book in a special table prepared for this purpose.

Calculation of the percentages of the results for each item of each standard according to the averages of the five-year Likert system. 


\subsection{Trust and Stability Analysis Tool}

Algebra units were analyzed in the books of the basic stage (third grade - fifth grade) in the light of peer analysis. Five teachers of mathematics were trained in the schools of the Hashemite Kingdom of Jordan. These steps were taken by each analyst individually, and the coefficient of the Cooper Agreement (1984) was calculated between one of the five researchers and analysts in the algebra criteria.

Table 2.

\begin{tabular}{c|c|c|c|c|c}
\hline Analyst & The first analyst & The second analyst & $\begin{array}{c}\text { The third } \\
\text { analyst }\end{array}$ & The fourth analyst & The fifth analyst \\
\hline Researcher & 0.84 & 0.86 & 0.77 & 0.89 & 0.79 \\
\hline
\end{tabular}

\subsection{Study Procedures}

The researcher carried out the following procedures to achieve the objectives of the study:

Access to theoretical literature and studies related to the standards of the National Council of Mathematics Teachers (NCTM, 2000), especially with regard to the standards of algebra content for the basic grade classes (3-5).

Develop the study tool based on the criteria of algebra content contained in the standards (NCTM, 2000) in line with the objectives of the study.

Presentation of the study tool to a group of arbitrators with the expertise and experience to verify the authenticity of the tool, and was verified the validity and consistency of analysis.

Perform the analysis of the units of algebra contained in each book of mathematics for grades (3-5).

Unloading the results of the analysis and statistical treatment in the appropriate ways, and discuss and interpret the results.

\subsection{Results and Discussion of the Study}

First: Results of the first question and discussed:

Text of the first question: What are the criteria for school mathematics issued by the National Council of Teachers of Mathematics (NCTM, 2000) on the content of algebra in math books in the third grade to the fifth grade in Jordan?

The answer to this question is the starting point for the rest of the study steps and procedures to reach the list of content standards to be available in algebra content in the basic stage math books. This list consisted of four main criteria of 14 sub-criteria, to the card to analyze the algebra content of mathematics textbooks the basic stage to see the availability of these standards in them.

Second: Results of the second question and discussed:

Question 2: What is the availability of school mathematics standards issued by the National Council of Mathematics Teachers (NCTM, 2000) on the content of algebra in math books in the third grade to the fifth grade in Jordan?

To answer this question, the researcher applied the content analysis tool prepared for this purpose, and analyzed the mathematics books for the third, fourth and fifth grades of the first and second books, which were applied in the academic year 2018/2019, and then the adoption of the arithmetic round to judge the availability The criteria in the study sample, and whether the degree of availability is very large or large, medium or low, and since the researcher was adopted in the analysis process on the scale of the five-year Licht; it was based on the averages on the following:

The average of (3.5-4) is available to a very large extent.

The average (2.5-3.49) is highly available.

The average (1.5-2.49) is available to a medium degree.

The average of $(0.5-1.49)$ is available to a small extent.

The average ( 0 - 0.49$)$ is not available.

Results of the first axis: Understanding relationships, patterns and functions

Describes generalizations about geometric and numerical patterns. 
Generalizations on engineering and numerical patterns.

Generalizations about geometric and numerical patterns.

Represent patterns and functions using words, tables and drawings.

Analyzes patterns and functions using words, tables and drawings.

Table 3. Mathematical averages of analyst estimates for each of the axis criteria Understanding relationships, patterns and functions

\begin{tabular}{ccccc}
\hline Class & the third & the fourth & Fifth & $\begin{array}{c}\text { General } \\
\text { average }\end{array}$ \\
\hline The first criterion & 0.6 & 0.9 & 1.4 & 0.96 \\
The second criterion & 0.4 & 0.9 & 1.6 & 0.96 \\
The third criterion & 0.3 & 0.8 & 1.1 & 0.73 \\
The fourth criterion & 0.2 & 0.7 & 1.96 & 0.95 \\
The fifth criterion & 0.1 & 1.2 & 1.47 & 0.92 \\
General average & 0.32 & 0.72 & 1.51 & 0.90 \\
\hline
\end{tabular}

It is clear from the data in Table 3 above that the overall average availability of standards in the first axis is 0.90 , which means that the degree of availability of the criterion for understanding the relationships, patterns and functions in the algebra content in the third, fourth and fifth grades was low. This indicates that the authors of the curriculum did not pay attention to this criterion, despite its importance in the foundation stage. This is also due to the nature of the content, which lacks training in patterns and functions and is sporadic in the preparation unit and because of the nature of the age of these classes. Table 3 also indicates that the criteria were distributed according to their degree of availability between the few and the middle by grade; the highest values were achieved in the fifth grade because their level of availability was reasonable. This is logical because this stage has a higher level of awareness and awareness than the previous two. "Describes generalizations about geometric and numerical patterns" and "defines generalizations of geometric and numerical patterns" more than other criteria, followed by the criterion "representing patterns and functions using words, tables and drawings".

The results also showed a general weakness in the understanding of relationships, patterns and functions in the third and fourth grades. The ratio of this criterion was $32 \%$. This result is consistent with the study of Mrayyan (2013), and differed with Rammona et al. (2015).

Results of the second axis: representation and analysis of mathematical positions and structures using algebraic symbols

Characterizes algebraic properties such as substitution, assembly and distribution.

Uses properties such as substitution, assembly and distribution in the calculations on the total numbers.

Represents the idea of the variable as anonymous value using letters or algebraic symbols.

Express mathematical relations using equations.

Table 4. Mathematical averages for analyst estimates for each of the axis criteria. Representation and analysis of mathematical positions, structures and algebraic symbols

\begin{tabular}{ccccc}
\hline Class & the third & the fourth & Fifth & $\begin{array}{c}\text { General } \\
\text { average }\end{array}$ \\
\hline The first criterion & 0.9 & 1.96 & 1.98 & 1.61 \\
The second criterion & 1.4 & 1.9 & 1.32 & 1.54 \\
The third criterion & 1.3 & 1.95 & 1.96 & 1.73 \\
The fourth criterion & 1.2 & 1.5 & 1.9 & 1.53 \\
General average & 1.2 & 1.83 & 1.79 & 1.60 \\
\hline
\end{tabular}

It is clear from the data in Table (4) above that the overall average availability of standards in the second axis is 1.60 , which means that the degree of availability of the criterion for the representation and analysis of mathematical positions and structures using algebraic symbols in algebra content in grades 3,4 and 5 was moderate. This corresponds to the nature of the Jordanian curriculum. The table also indicates that the 
availability of standards in grades 3, 4, and 5 was distributed in a medium and low degree. The criterion "represents the idea of the variable as unknown by using letters or algebraic symbols" with the highest availability of (1.73) this age range. While the criterion "characterizes algebraic characteristics such as substitution, assembly and distribution" was averaged 1.61, followed by the criterion "uses properties such as substitution, aggregation and distribution in calculations on whole numbers" and the criterion "expressing mathematical relationships using equations" (1.54) and (1.53). It is also noticed that the availability of these criteria in the fourth and fifth grade books is greater than in the third grade. This is logical due to the nature of the student's age and sports experience. In these two stages the student becomes more aware and has the ability to replace and assembly the distribution in computations compared to the third grade has little experience.

The results showed that there was a clear weakness in the criteria for the representation and analysis of mathematical positions and structures using the algebraic symbols of the third, fourth and fifth grades. This percentage was $40 \%$. This result is consistent with the study of Mrayyan (2013) and differed with Raba'a and Makadi (2016).

Results of the third axis: the use of mathematical models to represent and understand quantitative relationships

The positions of mathematical problems are integrated into things.

Uses representations such as drawings, tables and equations to draw the results.

Table 5. Mathematical averages of analyst estimates for each of the axis criteria use mathematical models to represent and understand quantitative relationships

\begin{tabular}{lllll}
\hline Class & the third & the fourth & Fifth & $\begin{array}{l}\text { General } \\
\text { average }\end{array}$ \\
\hline The first criterion & 0.8 & 1.9 & 2 & 1.56 \\
The second criterion & 0.5 & 1.89 & 2.5 & 1.63 \\
General average & 0.65 & 1.90 & 2.25 & 1.60 \\
\hline
\end{tabular}

It is clear from the data in Table 5 above that the overall mean availability of standards in the third axis is 1.60 , which means that the degree of availability of the criterion for the use of mathematical models to represent and understand quantitative relationships in algebra content in third, fourth and fifth grade textbooks was average. Table 5 also indicates that the criteria were broken down by grade, grade, and grade, and achieved the highest values in the fifth grade as they were highly available. The availability of the "uses such as graphs, tables and equations to derive results" The standard of "sports situations is mixed by things" to a medium extent, due to the nature of the content of algebra and its association with practical life.

There was a clear fluctuation in the availability of standards across the grades, as they were large in the fifth grade (2.25) and then became average in the fourth grade (1.90), and then became a few in a row as low as (0.65) This may be due to the cognitive backgrounds of analysts And the nature of their understanding of the standards of content Algebra, and in general results indicate that these standards are not considered in the curricula of the three grades significantly.

The results showed that there was a marked weakness in representation in the third, fourth and fifth grades. The ratio of this criterion was 39\%. This result is in line with the study of Mrayyan (2013) and differed with Rammona et al. (2015).

Results of the fourth axis: Analysis of change in different environments

Investigate the effect of change in one variable on the second variable.

Distinguish mathematical positions using constants or variables and comparison between them.

Describes mathematical attitudes using constants or variables and comparison between them. 
Table 6. The arithmetic averages of the analyst estimates for each of the axle standards analyze the change in different environments

\begin{tabular}{ccccc}
\hline Class & the third & the fourth & Fifth & $\begin{array}{c}\text { General } \\
\text { average }\end{array}$ \\
\hline The first criterion & 0.2 & 1.98 & 2.96 & 1.71 \\
The second criterion & 0.3 & 2.5 & 2.98 & 1.92 \\
The third criterion & 0.2 & 2.5 & 2.98 & 1.89 \\
General average & 0.23 & 2.32 & 2.97 & 1.84
\end{tabular}

It is clear from the data in Table 6 above that the overall average availability of standards in the third axis is 1.84 , which means that the degree of availability of the criterion of analysis of change in different environments in the algebra content of the third, fourth and fifth grades was moderate. Table 6 also shows that the criteria were broken down by grade between the few and the large by grade; the highest values were achieved in the fifth and fourth grade as they were highly available. The availability of a criterion "distinguishing mathematical attitudes using constants or variables and comparison between them" (1.92), followed by a criterion "describing mathematical attitudes using constants or variables and comparison between them" and a criterion that "explores the effect of change in one variable on the second variable" to a medium degree, due to the concentration of algebra content To distinguish irrigation positions Add and solve.

It is also noted that the weakest results across the three rows were the third grade, with the average availability of the criteria in its content $(0.23)$, which means that the parameters of change analysis are not available in the content of algebra. The highest results were in the fifth grade, with the average availability of standards in its content (2.97); this means a high degree of availability.

The results also showed a weakness in the analysis of the change in grades 3,4 and 5. The ratio of this criterion was 39\%. This result is in line with the results of the Raba'a and Makadi study (2016).

\section{Recommendations}

In light of the findings of the study, the researcher recommends the following:

Curriculum professionals should be familiar with international standards for curriculum development such as NCTM.

The need to review the weaknesses and poverty in mathematics books and strengthen the strengths.

Conduct more research and studies on the Jordanian curriculum by analyzing their content in the light of NCTM standards for all stages.

\section{References}

Abdelkader, Khalid. (2013). Difficulties in solving the verbal issue in mathematics among sixth graders in Gaza Governorate from the point of view of teachers. Al-Aqsa University Journal, 17(3): 77-106.

Abu Musa, Mufeed. (1997). Analysis of the developed mathematics for the fifth to eighth grades in Jordan in light of the international standards of the mathematics curriculum, unpublished master thesis, Yarmouk University, Irbid: Jordan.

Abu Al-Ajin, Ashraf. (2011). Evaluation of the content of Palestinian mathematics curricula in light of some NCTM standards. A magister message that is not published. Al-Azhar University - Gaza.

Al-Astal, Ibrahim. \& Rasheed, Samir. (2004). Adequate study planning for mathematics teachers. Educational Magazine, 18(7).

Al-Laqani, Ahmed. \& Aljammal, Ali. (2003). Glossary of educational educational terms in curricula and teaching methods. I. Cairo: World of the Book.

Jitendra, A., Griffin, C. \& Xin, Y. (2010). An Evaluation of Intented and Implemented Curriculas Adherence to the NCTM standards on Mathematics Achievement of Thid Grade Students. Journal of Curriculum and Instruction, 4(2): 33-50. https://doi.org/10.3776/joci.2010.v4n2p33-50

Mrayyan, S. (2013). Jordanian Elementary Math Curriculum and Geometry Content Along with National Coucil Teachers of Mathematics (NCTM) Grades (1-6) as Case Study. Greener Journal of Educational Research, 3(3): 144-154. https://doi.org/10.15580/GJER.2013.3.021313452 
NCTM (1989). Curriculum and evahuation standards for school Mathematics. Reston, VA.: National Council of Teacher of Mathematics.

NCTM (1991). Professional standards for teaching mathematics, VA: National Council of Teacher of Mathematics.

NCTM (1995). Assessment standards for school mathematics, VA: National Council of Teacher of Mathematics.

NCTM (2000). Principles and standards for school mathematics, VA: National Council of Teacher of Mathematics.

Obaid, William. (2004). Teaching mathematics to all children in light of the requirements of standards and culture of thinking. Amman: Dar Al Masirah for Publishing and Distribution.

Rammna, Modern., Apollo, Khaled., Al-Hayat, Mohammed. \& Alkrimin, Raed. (2015). Analysis of the content of the measurement according to the standards (NCTM, 2000) of mathematical operations in mathematics books the basic stage from the first to fourth grade in Jordan. Journal of Palestine University for Research and Studies, 5(2).

Sadiq, Ismail. (2001). Methods of Teaching Mathematics / Theories and Applications, Reference Series in Education and Psychology, 1, Cairo: Arab Thought House.

Shahry, Abdullah. (2015). Evaluation of the content of mathematics book for the first grade secondary in Saudi Arabia in the light of NCTM standards. Arab Studies in Education and Psychology - Saudi Arabia, (68): 191-220.

Zubi, Ali. \& Al-Obaidan, Abdullah. (2014). Analysis of the mathematics book for the fourth grade in Saudi Arabia in the light of NCTM standards. Educational Sciences, 41(1): $317-332$. https://doi.org/10.12816/0007881

\section{Copyrights}

Copyright for this article is retained by the author(s), with first publication rights granted to the journal.

This is an open-access article distributed under the terms and conditions of the Creative Commons Attribution license (http://creativecommons.org/licenses/by/4.0/). 